\title{
CHEMOTHERAPY
}

\section{After 25 years, therapy for poor-prognosis GCTs advances}

Most patients $(>80 \%)$ with germ-cell tumours (GCTs), the most-common cancer in males aged 15-24 years, are cured. However, $~ 50 \%$ of patients with high-risk disease who receive the standard four cycles of bleomycin, etoposide and cisplatin (BEP) chemotherapy do not survive beyond 5 years. New data suggest that a personalized chemotherapy approach could improve outcomes in this subgroup.

Previously, Karim Fizazi et al. showed that reduced blood tumour marker levels after 3 weeks of chemotherapy identified the patients with poor-prognosis GCTs at most risk of treatment failure. Thus, in a recent phase III trial, Fizazi et al. stratified patients into favourable and unfavourable cohorts based on their response to one $\mathrm{BEP}$ cycle. Patients with an unfavourable response were randomly assigned to receive continued $\mathrm{BEP}$ or dose-dense chemotherapy comprising BEP, paclitaxel, oxaliplatin, ifosfamide and mesna with granulocyte-colony stimulating factor support. "Our hypothesis was that early intensification could improve outcomes for these hard-to-treat patients," explains Fizazi. The standard BEP regimen was continued in the favourable group.

For the unfavourable groups, dose-dense therapy improved 3-year progression-free survival (PFS) compared with standard BEP (59\% versus 48\%). "This is the first time in 25 years that an improvement in outcome has been demonstrated for this group of patients," states Fizazi. "We also demonstrated that cure is achieved with classic chemotherapy for most patients

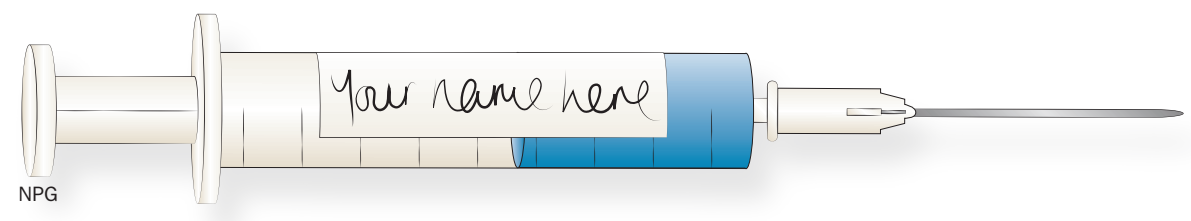

with a favourable response." Indeed, among these patients the 3-year PFS rate was 70\%. Although not significantly different, the 3 -year overall survival rates were $73 \%$ and $65 \%$ in the unfavourable dose-dense and BEP cohorts, respectively, and $84 \%$ in the favourable group.

"Thanks to this study, we can better personalize therapy for patients with a poor prognosis: four cycles of BEP for patients with initial favourable declines in levels of tumour markers, switching to the dose-dense intensified regimen for those with an unfavourable decline," concludes Fizazi. However, the dose-dense regimen was complex and challenging with regards to administration and toxicity, and probably needs further optimization.

David Killock
Original article Fizazi, K. et al. Personalised chemotherapy based on tumour marker decline in poor prognosis germ-cell tumours (GETUG 13): a phase 3, multicentre, randomised trial. Lancet Oncol. doi:10.1016/S1470-2045(14)70490-5 